\title{
Characteristic Temperatures of Waxy Crude Oils
}

\author{
Zhu Yingru, Zhang Jinjun, Li Hongying and Chen Jun \\ (Beijing Key Laboratory of Urban Oil and Gas Distribution Technology, China University of Petroleum, Beijing 102249, China)
}

\begin{abstract}
Gel point or pour point is widely used to evaluate the low temperature flowability of crude oil. However, it is not adequate to describe the gelling properties of waxy crude oils under flow conditions with gel point or pour point, since the rheological behavior of crude oils is dependent on shear history. Waxy crude oils tend to gel at a low temperature. Based on gelation theory, the characteristic temperature of waxy crude oil was determined by analyzing viscosity-temperature data. Two mathematical models were developed for calculating characteristic temperatures of virgin crude oils and pour point depressants (PPD) beneficiated crude oils, respectively. By using these two models, the characteristic temperatures of crude oils that have experienced shearing and thermal histories can be predicted. The model for predicting the characteristic temperature of virgin crude oils has an average relative deviation of $4.5 \%$, and all predicted values have a deviation within $2{ }^{\circ} \mathrm{C}$. Tested by 42 sets of data, the prediction model for crude oil treated with PPD has a high accuracy, with an average relative deviation of $4.2 \%$, and 95.2 percent of predicted values have a deviation within $2{ }^{\circ} \mathrm{C}$. These two models provide useful ways for predicting the flowability of crude oils in pipelines when only wax content, wax appearance point and gel point are available.
\end{abstract}

Key words: Waxy crude, low temperature flow ability, characteristic temperature, mathematical model

\section{Introduction}

Many crude oils contain significant quantities of wax, which can crystallize during oil production, transportation and storage, resulting in an increase in oil viscosity. This may cause some difficulties in the transport of crude oil in pipelines. At present, thermal and chemical treatments, especially pour point depressant (PPD) treatment, are widely used in the transportation of waxy crude oils. As is known to all, the flowability of waxy crude oils, especially the PPDbeneficiated waxy crude oils, are greatly influenced by thermal and shear histories during pipeline transportation (Zhang, et al., 1993; 2001; 2004; Zhang, 2002). The effect of shear on flow behavior of waxy crude oils during cooling was systematically investigated by Wardhaugh and Boger (1991) and Wardhaugh, et al. (1998). Chen and his coworker $(2003 ; 2005)$ proposed the concept of characteristic temperature of waxy crude oils, which is the temperature corresponding to the maximum curvature of the non-dimensional viscosity-temperature curve. Experimental results have shown that the characteristic temperature varies linearly with shear rate and cooling rate (Chen, 2003; Chen, et al., 2005). Characteristic temperature can be used as a factor to evaluate low temperature flowability of waxy crude oils with different shear and thermal histories. Li and her coworkers (2005) discovered that the gelation temperature almost coincides with the characteristic temperature at a given dynamic stress. For rather low stress, the formation of wax crystal network will not be disturbed, and the crude is gelled as in quiescent conditions, both gelation temperature and characteristic temperature are close to the gel point of the specimen.

In this paper, viscosity-temperature dependence was measured by temperature scanning experiments in a coaxial cylinder viscometer. On the basis of gelation theory, the characteristic temperature of waxy crude oil was determined by using regular data analysis. The value of the characteristic temperature obtained by this method is close to that obtained by the non-dimensional curvature method. Semi-empirical equations between characteristic temperature and viscous entropy generation in the shear process were developed.

\section{Experimental}

\subsection{Experimental apparatus and oil samples}

Six virgin waxy crude oils and four PPD-beneficiated crude oils were investigated in this work. Their main physical properties are listed in Table 1. In order to study the flow behavior of waxy crude oils with various thermal and shear histories, the viscosity-temperature dependency was investigated by temperature scanning experiments performed in a rotational viscometer. The experimental apparatus was a coaxial cylinder sensor system MV-DIN of HAAKE Viscometer 550, equipped with a HAAKE C25P cooling bath.

\subsection{Experimental procedures}

All oil samples were heated to $80{ }^{\circ} \mathrm{C}$ for $2 \mathrm{~h}$ to eliminate previous thermal and shear histories, and cooled quiescently to room temperature and kept at this temperature for at least $48 \mathrm{~h}$ before use. Experiments were conducted as follows. 1) The sample was heated to a specified temperature for $30 \mathrm{~min}$, and then put into the coaxial cylinder system which is preheated to the same 
temperature. 2) After $10 \mathrm{~min}$, the sample was cooled at a given cooling rate and at the same time the cylinder sensor was rotated at a given shear rate. The viscosity and temperature data were recorded simultaneously during cooling. Therefore a viscosity-temperature curve for the temperature scanning experiment could be obtained.

Table 1 Physical propert ies of waxy crude oil samples

\begin{tabular}{ccccccc}
\hline No. & $\begin{array}{c}\text { Density at 20 } \\
\mathrm{kg} / \mathrm{m}^{3}\end{array}$ & $\begin{array}{c}\text { Wax content } \\
\text { wt.\% }\end{array}$ & $\begin{array}{c}\text { Wax appearance temperature (WAT) } \\
{ }^{\circ} \mathrm{C}\end{array}$ & $\begin{array}{c}\text { Pour point } \\
{ }^{\circ} \mathrm{C}\end{array}$ & $\begin{array}{c}\text { Gel point } \\
{ }^{\circ} \mathrm{C}\end{array}$ & Notes \\
\hline 1 & 858 & 22.9 & 44.0 & 36 & 34 & Virgin \\
$1-1$ & 858 & 22.9 & 44.0 & 28 & 25 & PPD-beneficiated \\
\hline 2 & 849 & 22.5 & 49.9 & 35 & 32 & Virgin \\
$2-1$ & 849 & 22.5 & 49.9 & 28 & 25 & PPD-beneficiated \\
$2-2$ & 849 & 22.5 & 49.9 & 25 & 22 & PPD-beneficiated \\
\hline 3 & 850 & 20.3 & 57.8 & 35 & 33 & Virgin \\
4 & 892 & 22.1 & 46.5 & 35 & 32 & Virgin \\
5 & 801 & 10.2 & 21.5 & 18 & 16 & Virgin \\
\hline 6 & 859 & 19.7 & 37.0 & 36 & 33 & Virgin \\
$6-1$ & 866 & 15.0 & 42.0 & 22 & 20 & PPD-beneficiated \\
\hline
\end{tabular}

\section{Results and discussion}

\subsection{Measured characteristic temperatures of waxy crude oils}

The equation of viscosity vs. temperature for temperature scanning experiments can be described as follows.

$$
\mu=A \exp \left(\frac{E_{\mathrm{a}}}{R T}\right)
$$

where $\mu$ is the apparent viscosity, $\mathrm{Pa} \cdot \mathrm{s} ; A$ is the pre-exponential factor or frequency factor, $\mathrm{Pa} \cdot \mathrm{s} ; E_{\mathrm{a}}$ is the activation energy, $\mathrm{J} / \mathrm{mol} ; R$ is the universal gas constant; and $T$ is the absolute temperature, $\mathrm{K}$.

The waxy crude oil tends to gel with increasing activation energy of gelation, so it is necessary to analyze the gelation process of waxy crude oil, and then to determine the maximum activation energy of gelation, $E_{\max }$ and its corresponding temperature, i.e. the characteristic temperature.

Data processing procedures used were as follows: 1) The measured viscosity-temperature curve $\mu-T$ of oil sample was transformed into the natural logarithm of viscosity $\ln \mu$ vs. the reciprocal of absolute temperature $1 / T$ curve. 2) The transformed curve was plotted as a broken line graph consisting of a number of straight-line segments. 3) The slope of each straight-line segment was calculated. 4) The maximum slope was determined for these straight-line segments and its corresponding initial temperature was the characteristic temperature.

Several characteristic temperatures measured under different experimental conditions are listed in Table 2.

The characteristic temperature of virgin crude oil closely approximates its pour point under different experimental conditions, while the characteristic temperature of PPD-beneficiated crude oil is much lower than its pour point when the entropy generation in the oil system is low (see Table 2).

\subsection{Viscous entropy generation during shearing}

In thermodynamics, entropy generation, also called entropy production, is a measure of irreversibility of a process. The viscous entropy generation can be calculated from the following equation (Zhang, et al., 2004):

$$
s_{\mathrm{g}}=\frac{\Phi}{\rho T}
$$

where $s_{\mathrm{g}}$ is the entropy generation per unit mass of viscous fluid, $\mathrm{J} /(\mathrm{kg} \cdot \mathrm{K}) ; \Phi$ is the energy dissipation per unit volume of viscous fluid, $\mathrm{J} / \mathrm{m}^{3} ; \rho$ is the density of crude oil, $\mathrm{kg} / \mathrm{m}^{3}$.

For Newtonian fluids, the energy dissipation in viscous flow is:

$$
\Phi=\mu \dot{\gamma}^{2} t
$$

For power law fluids, the energy dissipation in viscous flow is: 
Table 2 Characteristic temperatures and entropy generation values at different shear rates and cooling rates

\begin{tabular}{|c|c|c|c|c|c|c|c|}
\hline \multirow{2}{*}{$\begin{array}{l}\text { Cooling rate } \\
{ }^{\circ} \mathrm{C} / \mathrm{min}\end{array}$} & \multirow{2}{*}{$\begin{array}{c}\text { Shear rate } \\
\mathrm{s}^{-1}\end{array}$} & \multicolumn{2}{|c|}{ Entropy generation, $\mathrm{J} /(\mathrm{kg} \cdot \mathrm{K})$} & \multicolumn{4}{|c|}{ Characteristic temperature, ${ }^{\circ} \mathrm{C}$} \\
\hline & & No. 2 & No. $2-2$ & No. 2 & No. 2-2 & No. 1 & No. $1-1$ \\
\hline 0.05 & 125 & 24.16 & 49.22 & 34.9 & 26.5 & - & - \\
\hline 0.05 & 100 & - & - & - & - & 36.2 & - \\
\hline 0.1 & 125 & 10.69 & 21.82 & 34.3 & 24.1 & - & - \\
\hline 0.2 & 125 & 5.27 & 10.51 & 33.7 & 22.7 & 36.0 & 27.5 \\
\hline 0.2 & 75 & - & - & - & - & 35.1 & 25.4 \\
\hline 0.2 & 60 & - & - & - & - & 34.7 & 24.6 \\
\hline 0.2 & 30 & - & - & - & - & 34.5 & 21.5 \\
\hline 0.3 & 125 & 3.28 & 6.68 & 33.5 & 20.8 & - & - \\
\hline 0.3 & 100 & - & - & - & - & 35.1 & - \\
\hline 0.4 & 150 & - & 7.12 & - & 21.3 & - & - \\
\hline 0.4 & 125 & 2.5 & 4.89 & 33.4 & 20.0 & - & - \\
\hline 0.4 & 100 & 1.54 & 3.02 & 32.8 & 18.1 & 35.1 & 23.9 \\
\hline 0.4 & 75 & 0.85 & 1.81 & 32.5 & 16.0 & - & - \\
\hline 0.4 & 60 & 0.58 & 1.11 & 31.9 & 14.9 & - & - \\
\hline 0.4 & 50 & 0.38 & 0.81 & 31.2 & 14.1 & - & 20.5 \\
\hline 0.4 & 40 & - & 0.54 & - & 12.4 & - & - \\
\hline 0.4 & 25 & 0.07 & - & 30.8 & - & - & 20.2 \\
\hline
\end{tabular}

$$
\Phi=K \dot{\gamma}^{n+1} t
$$

where $\dot{\gamma}$ is the shear rate, $\mathrm{s}^{-1} ; t$ is the shear time, $\mathrm{s} ; n$ is the rheological behavior index; and $K$ is the consistency coefficient, $\mathrm{Pa} \cdot \mathrm{s}^{n}$.

For the cooling process accompanied with shearing, the temperature range can be divided into a certain number of small intervals. The entropy generation of each interval can be written as Eq. (5).

$$
\Delta s_{\mathrm{g}, i}=\frac{\Phi}{\rho_{i} T_{i}}
$$

The total viscous entropy generation is the sum of those produced in each small interval.

$$
s_{\mathrm{g}}=\sum_{i=1}^{n} \frac{\Phi_{i}}{\rho_{i} \Delta T_{i}}
$$

The above equations indicate that the entropy generation in viscous fluid flow is related to shear rate and shear time. In the temperature scanning experiments, if the shear rate and temperature range of cooling are identical, the shear time prolongs with the reduction of cooling rate, therefore entropy generation increases. The lower the cooling rate, the greater the entropy generation in viscous fluid flow. Cooling rate and shear rate have great influences on the entropy generation in viscous fluid flow. When shear rate is $125 \mathrm{~s}^{-1}$, the curves of entropy generation vs. cooling rate for virgin crude oil (No. 2) and PPD-beneficiated crude oil (No. 2-2) are shown in Fig. 1. The influence of shear rate on the entropy generation in viscous fluid flow is shown in Fig. 2.

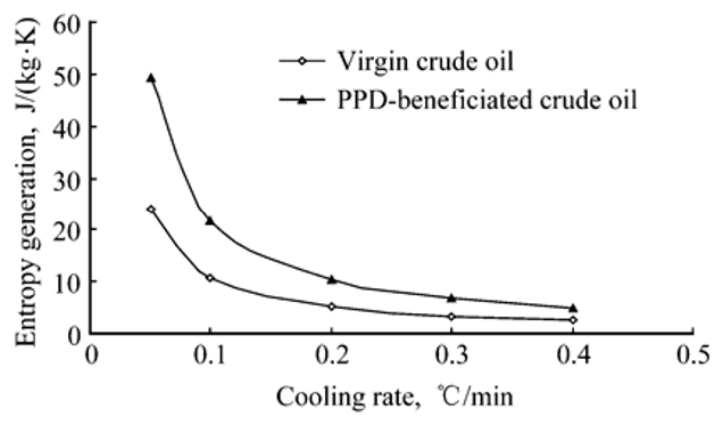

Fig. 1 Curves of entropy generation vs. cooling rate

The entropy generation values calculated from above-mentioned equations for some oil samples are also listed in Table 2.

Curves of characteristic temperature vs. viscous entropy generation during shearing for virgin oil sample (No. 2) and PPD-beneficiated oil sample (No. 2-2) are shown in Fig. 3. This indicates that the characteristic temperature increases with increasing viscous entropy generation under experimental conditions. Fig. 3 also 
indicates that the PPD-beneficiated crude oil has a relatively broader variation in characteristic temperature compared with the virgin oil. This is because the size of wax crystals dispersing separately in beneficiated oil is larger than that in the virgin waxy crude oil, which results in the reduction of pour point of oil, these big wax crystals are thereby more vulnerable to shearing and cooling and transform into tiny wax crystals. This leads to the formation of wax network structures with a high strength at lower temperatures, the flowability thereby becomes worse.

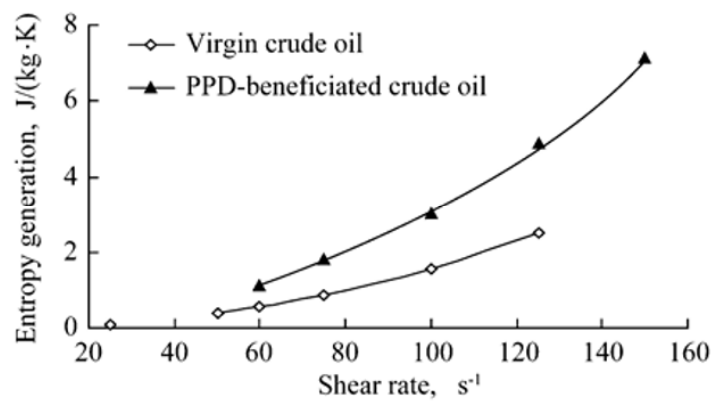

Fig. 2 Curves of entropy generation vs. shear rate

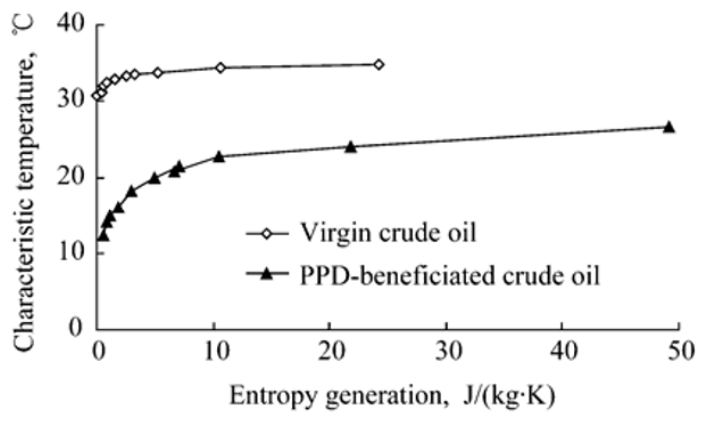

Fig. 3 Characteristic temperature vs. viscous entropy generation for virgin oil sample (No. 2) and PPD-beneficiated oil sample (No. 2-2)

\subsection{Mathematical model for characteristic temperatures of virgin crude oils}

In the experiments, 6 virgin crude oils were sheared at given shear rates and cooling rates. The shear rates are $25,40,50,60,75,100,125$ and $150 \mathrm{~s}^{-1}$, respectively. The cooling rates of oil sample for measurements are $0.05,0.1,0.2,0.3$ and $0.4{ }^{\circ} \mathrm{C} / \mathrm{min}$, respectively.

In order to develop a universal mathematical model for calculating the characteristic temperature of virgin crude oils, a new variable $R_{\mathrm{c}}$, relative characteristic temperature, was introduced when processing experimental data. The relative characteristic temperature can be written as follows:

$$
R_{\mathrm{c}}=\frac{T_{\mathrm{w}}-T_{\mathrm{c}}}{T_{\mathrm{w}}-T_{\mathrm{g}}}
$$

where $T_{\mathrm{c}}$ is the characteristic temperature; $T_{\mathrm{w}}$ is the wax appearance temperature (WAT); and $T_{\mathrm{g}}$ is the gel point of virgin crude oil.

The correlation between relative characteristic temperature and entropy generation for different virgin crude oils was analyzed and curves between $R_{\mathrm{c}}$ and $s_{\mathrm{g}}$ are shown in Fig. 4. This indicates that the relative characteristic temperature of a virgin crude oil decreases with increasing viscous entropy generation. When viscous entropy generation is less than a specific value, relative characteristic temperature decreases sharply as viscous entropy generation increases. And relative characteristic temperature $R_{\mathrm{c}}$ decreases gently and tends to be constant with an increase in viscous entropy generation.

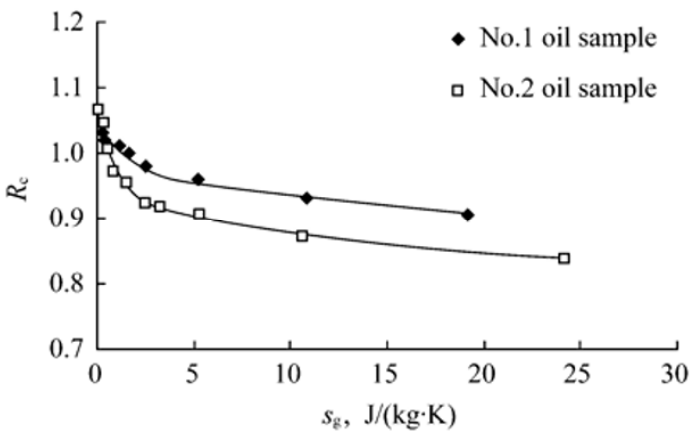

Fig. 4 Relative characteristic temperature vs. viscous entropy generation for virgin oil samples

As shown in Fig. 4, the correlation between relative characteristic temperature and viscous entropy generation for virgin crude oils can be expressed as follows:

$$
R_{\mathrm{c}}=\ln \left(a s_{\mathrm{g}}^{b}\right)
$$

The values of coefficients $a$ and $b$ in Eq. (8) are different for different virgin crude oils. Therefore, wax content $\left(c_{\mathrm{W}}\right)$ is introduced, assuming the following equation could describe the correlation between relative characteristic temperature and viscous entropy generation:

$$
R_{\mathrm{c}}=\ln \left(k_{0} s_{\mathrm{g}}^{k_{1}} c_{\mathrm{w}}^{k_{2}}\right)
$$

Eq. (9) indicates that relative characteristic temperature is related to wax content and viscous entropy generation during shearing.

Wax content $\left(c_{\mathrm{w}}\right)$ and viscous entropy generation $\left(s_{\mathrm{g}}\right)$ are independent variables, and relative characteristic temperature $\left(R_{\mathrm{c}}\right)$ is a dependent variable. Set $X_{0}=\ln \left(k_{0}\right)$, $X_{1}=\ln s_{\mathrm{g}}, X_{2}=\ln c_{\mathrm{w}}$ and $Y=R_{\mathrm{c}}$, then Eq. (9) can be transformed as follows:

$$
Y=X_{0}+k_{1} X_{1}+k_{2} X_{2}
$$


Fifty sets of experimental data on 6 virgin crude oils were used to regress the coefficients $X_{0}\left(\ln k_{0}\right), k_{1}$ and $k_{2}$ with the least squares method and then substituting these values into Eq. (9) gives

$$
R_{\mathrm{c}}=\ln \left(0.9998 s_{\mathrm{g}}^{-0.05414} c_{\mathrm{w}}^{0.31335}\right)
$$

The above equation can be expressed in the following form:

$$
R_{\mathrm{c}}=-0.05414 \ln s_{\mathrm{g}}+0.31335 \ln c_{\mathrm{w}}-0.00019
$$

Substituting Eq. (7) into Eq. (12) gives:

$$
T_{\mathrm{c}}=T_{\mathrm{w}}-\left(T_{\mathrm{w}}-T_{\mathrm{g}}\right)\left(-0.05414 \ln s_{\mathrm{g}}+0.3133 \ln c_{\mathrm{w}}-0.00019\right)
$$

The hypothetical model (Eq. (9)) can be deduced by analyzing experimental data. It is necessary to do sensitivity analysis for model validation. An $F$-test is often used to test the hypothetical model in mathematical statistics.

If $F>F_{1-a}(p, n-p-1)$, the hypothetical model is correct, otherwise, it is wrong.

For Eq. (10), $p=2, n=50$

$$
F=\frac{\left(\sum_{i=1}^{n}\left(\hat{y}_{i}-\bar{y}\right)^{2}\right) / p}{\left(\sum_{i=1}^{n}\left(y_{i}-\hat{y}\right)^{2}\right) /(n-p-1)}=25.10
$$

$F_{1-a}(p, n-p-1)=F_{0.99}(2,47)=5.09$ according to $F$ distribution table. There is an obvious linear relationship between dependent variable $(Y)$ and independent variables $\left(X_{1}, X_{2}\right)$, because $F$ is greater than $F_{0.99}(4,47)$. As a result, the hypothetical model can be used to describe the relationship between independent variables and dependent variable.

For 50 sets of experimental data, the prediction equation has high accuracy for predicting characteristic temperature, with an average relative deviation of $4.5 \%$, and $100 \%$ of predicted values have a deviation within $2{ }^{\circ} \mathrm{C}$ (see Fig. 5). By using Eq. (13), the characteristic temperature of virgin crude oil with different changes in entropy generation can be predicted when only WAT, gel point and wax content are available.

\subsection{Mathematical model for characteristic temperatures of PPD-beneficiated crude oils}

Four PPD-beneficiated crude oils were sheared at given shear rates and cooling rates and 42 sets of experimental data were acquired. The experimental conditions are identical to the ones specified in the temperature scanning experiments of virgin crude oils. Experimental results show that characteristic temperatures of beneficiated oils are related to the wax appearance temperature and gel point. The model for calculating characteristic temperature of beneficiated crude oils was obtained by using the same regression analysis.

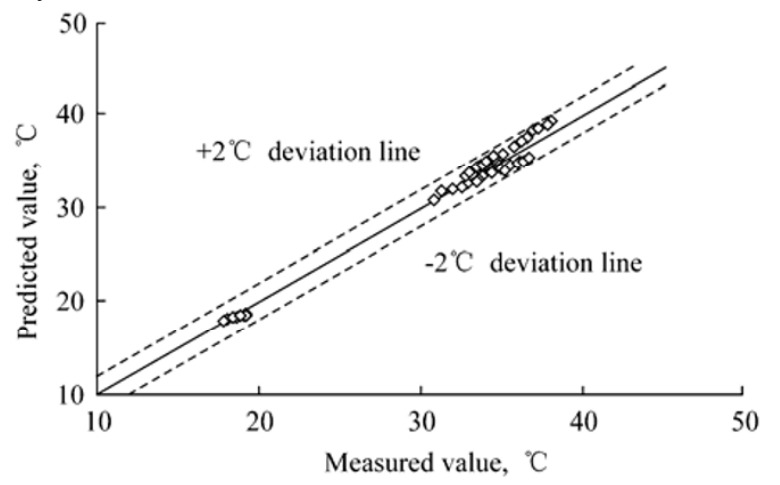

Fig. 5 Comparison of characteristic temperatures predicted from Eq. (13) with the measured data for virgin crude oils

$$
T_{\mathrm{c}}=T_{\mathrm{w}}-\left(T_{\mathrm{w}}-T_{\mathrm{g}, 0}\right)\left(-0.1172 \ln s_{\mathrm{g}}+0.2286 \ln c_{\mathrm{w}}+0.6127\right)
$$

where $T_{\mathrm{g}, 0}$ is the gel point of PPD-beneficiated crude oil before shear.

Eq. (14) has high accuracy, with an average relative deviation of $4.2 \%$ and $95.2 \%$ of predicted results having a deviation within $2{ }^{\circ} \mathrm{C}$ (see Fig. 6).

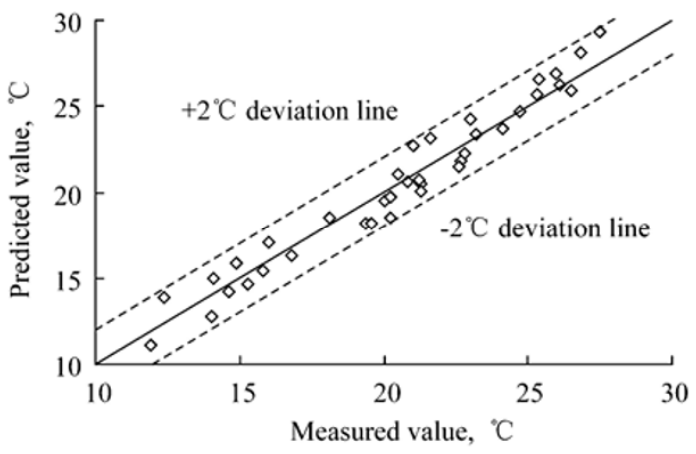

Fig. 6 Comparison of characteristic temperatures predicted from Eq. (14) with the measured data for PPD-beneficiated crude oils

\section{Conclusions}

1) The characteristic temperature of waxy crude oil, corresponding to the maximum value of gelling activation energy, can be determined by regular data analysis of viscosity-temperature dependency.

2) The characteristic temperature of virgin crude oil closely approximates its pour point under different experimental conditions. While the characteristic temperature of PPD-beneficiated crude oil is much lower than its pour point when the entropy generation in the oil system is low. Characteristic temperature can be 
used to evaluate the flowability of waxy crude oils with different shear and thermal histories.

3) Mathematical models for predicting characteristic temperatures of virgin crude oils and PPD-beneficiated crude oils are developed. The characteristic temperatures of waxy crude oils that have experienced shear and thermal histories can be predicted with these two models. The predicted values are very close to the measured results.

\section{Acknowledgements}

This paper was supported by the Youth Innovation Fund of China National Petroleum Corporation (CNPC).

\section{References}

Chen J. (2003) Flow Characteristics and Pipelining Characteristics of Waxy Crude Oils. PhD Thesis. Beijing: China University of Petroleum (in Chinese)

Chen J., Zhang J. J. and Li H. Y. (2005) A study of low-temperature characteristics of waxy crude oils. In: Luo Y. S., Rao Q. and Xu Y. (eds) Advances in Rheology and Its Applications. USA Inc: Science Press. 917-920

Li H. Y. Zhang J. J. and Chen J. (2005) Gelling Properties of Waxy Crudes in Quiescent and Under Shearing Condition. In: Luo Y. S., Rao Q. and Xu Y. (eds) Advances in Rheology and Its Applications. USA Inc: Science Press. 921-923

Waudhaugh L. T., Boger D. V. and Tonner S. P. (1988) Rheology of waxy crude oils. International Meeting on Petroleum Engineering held in Tianjin, China, 1-4 November 1988. SPE paper 17625

Wardhaugh L. T. and Boger D. V. (1991) Flow characteristics of waxy crude oils: Application to pipeline design. AIChE Journal, 37(6), 871-885

Zhang F. S., Xie H. Z., and Dong L. J. (2001) A study of BEM series of pour point depressants and their application in China. SPE International Symposium on Oilfield Chemistry held in Houston, Texas, 13-16 February 2001. SPE paper 65381

Zhang J. J., Zhao T. H., Liu Z. H. and Huang Q. Y. (1993) Shear effect on rheological characteristics of Xinjiang mixed oils treated with pour point depressant. Journal of the University of Petroleum, China, 17(6), 74-78 (in Chinese)

Zhang J. J. (2002) Technologies for pipelining high pour point and viscous crude oils and their development. Engineering Science, 4(6), 71-76 (in Chinese)

Zhang J. J., Pan D. L., Tu H. M., Huang Q. Y. and Zhang F. (2004) A mathematical model for shear effect of gel point crude beneficiated with pour point depressants. Acta Petrolei Sinica, 52(2), 96-99 (in Chinese)

\section{About the first author}

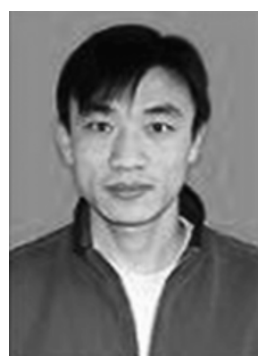

Zhu Yingru was born in 1977 and received his BA degree in 2001 from the Daqing Petroleum Institute. Now he is a PhD candidate in the School of Petroleum Engineering, China University of Petroleum (Beijing), with his research interests in petroleum engineering.

E-mail: zhuyingru@163.com

(Received December 6, 2006) (Edited by Sun Yanhua) 